\title{
Combined hamartoma of the retina and retinal pigment epithelium in a 6 -year-old girl — a case report
}

\author{
Dorota Borowicz', Katarzyna Biela', Ewa Teresińska', Tomasz Bentkowski', Waldemar Kędziora', \\ Katarzyna Nowomiejska $\odot^{2}$, Robert Rejdak $\oplus^{2}$ \\ 'Pope John Paul II Independent Public Provincial Hospital, Zamość, Poland \\ ${ }^{2}$ Department of General Ophthalmology, Medical University of Lublin, Lublin, Poland
}

\begin{abstract}
Combined hamartoma of the retina and retinal pigment epithelium (CHRRPE) is a rare, benign, usually onesided disease that can cause significant painless vision loss. Combined hamartoma of the retina and retinal pigment epithelium is most commonly found in isolated forms, but reports are also available describing compounds with neurofibromatosis types 1 and 2. CHRRPE has increased retinal pigmentation, tortuous vessels, and the presence of epiretinal membranes.

In our article, we present a case report of a 6-year-old girl who was diagnosed with CHRRPE.
\end{abstract}

KEY WORDS: hamartoma of the retina and retinal pigment epithelium

Ophthalmol J 2020; Vol. 5, 42-45

\section{INTRODUCTION}

Combined hamartoma of the retina and retinal pigment epithelium (CHRRPE) is a rare, benign, usually one-sided tumor that can cause significant painless vision loss and strabismus. Combined hamartoma of the retina and retinal pigment epithelium is most commonly found in isolated forms, but reports are also available describing compounds with neurofibromatosis types 1 and 2. The classic clinical symptoms of CHRRPE increased retinal pigmentation, tortuous vessels, and the presence of epiretinal membranes. The diagnosis of CHRRPE is established by combining classic clinical signs with diagnostic features on ultrasonography (noncalcified mass) and pleated, thickened retina with disorganized of the photoreceptor on optical coherence tomography (OCT).
This tumor can resemble choroidal melanoma or retinoblastoma.

We report a case of CHRRPE with poor visual acuity and strabismus.

\section{CASE REPORT}

A 6-year-old girl was admitted to the Ophthalmological Emergency Room due to slow, painless decrease of visual acuity in the left eye and left eye esotropia. The mother of the girl did not notice the beginning of the deterioration of vision and the incorrect position of the left eye. Parents denied the injury, the child's general health was normal, the girl was born without complications at delivery. On exam, the best-corrected visual acuity was $20 / 20$ OD and 20/250 OS. The anterior seg- 


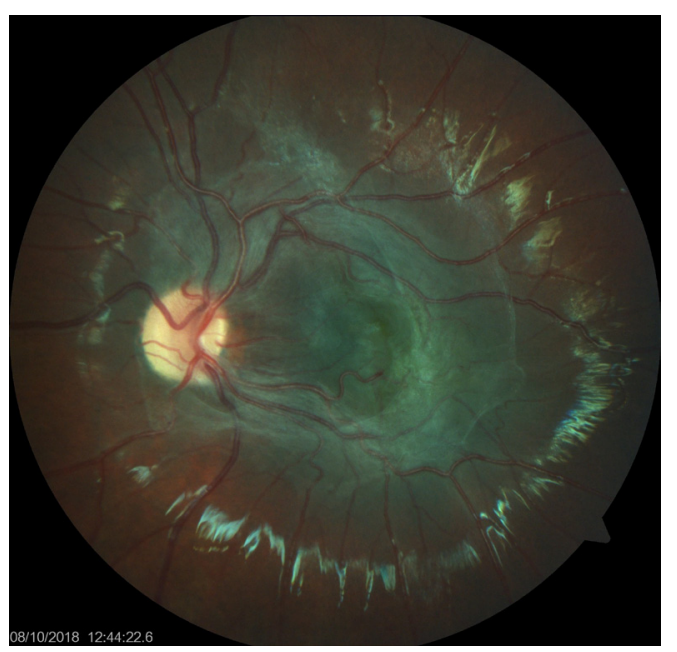

FIGURE 1. In the fundoscopy of the left eye, an area of increased pigmentation of the retinal pigment epithelium was observed, vascular arcades were distorted and shifted towards the macula. Within the lesion a shimmering gray epiretinal membrane was visible

ment was unremarkable OU. In the fundoscopy of the left eye, an area of increased pigmentation of the retinal pigment epithelium was observed, vascular arcades were distorted and shifted towards the macula. Within the lesion a shimmering gray epiretinal membrane was visible. The retinal periphery was normal (Fig. 1). There wasno deviation in the fundoscopy of the right eye. Optical coher- ent tomography of the left eye showed a significant thickening of the retina with hyporeflective shading of normal retinal layers and with the presence of the epiretinal membrane. The right eye OCT was normal (Fig. 2). Type B ultrasound showed a lobular lesion in the macula region with a calcified plaque on the lesion surface. The patient was referred to the Ophthalmological Oncology Clinic in Cracow where the diagnosis was confirmed based on a clinical examination and additional tests, i.e. OCT, ultrasound. The patient remains under constant ophthalmological control, her condition is stable.

\section{DISCUSSION}

Embryologically, the retinal pigment epithelium (RPE) develops from the outer layer of the optic cup - the ectoderm, and the neurosensory retina from the inner layer. In the inner layer, the cells adherent to the intraretinal space differentiates into photoreceptors and the cells of the next layer form Muller cells as well as bipolar cells and ganglion cell axons. Retinal and pigmented epithelial hamartoma is, therefore, a congenital lesion in which the developmental disorders of all separately developing retinal layers occurs $[1,2]$.

Histologically, CHRRPE consists of pigmented, glial and vascular cells in various propor-

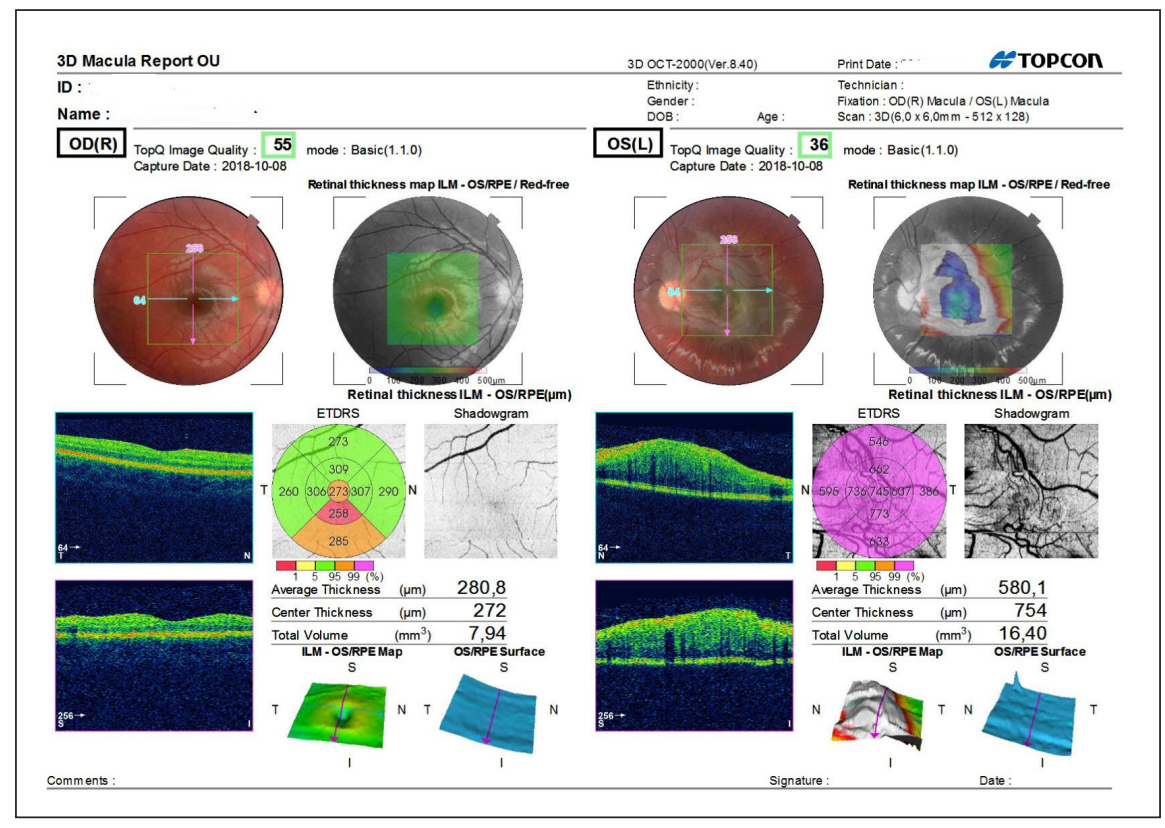

FIGURE 2. Optical coherent tomography (OCT) of the left eye showed a significant thickening of the retina with hyporeflective shading of normal retinal layers and with the presence of the epiretinal membrane. The right eye OCT was normal 
tions. It is most often located near the optic nerve $(76 \%)$, macula $(17 \%)$ and in the peripheral retina (17\%) [3].

Gass was the first to use the term retinal hamartoma and RPE in 1973. He was the first to describe clinical features of CHRRRPE as:

— slightly elevated dark gray lesion affecting the retina, RPE and surrounding vitreous;

- expansion towards the peripher;

- connecting to the neighboring RPE;

— surrounded by dense gray-white retina;

- showing internal surface compression;

- without peripheral RPE and choroidal atrophy;

- no retinal detachment, haemorrhage, vitreous effusion [4].

According to Gass, CHRRPE was initially divided into two subcategories depending on the presence or absence of involvement of the optic disc. [5] These two types of CHRRPE have slightly different histological features. CHRRPE, which does not include the optic disc, shows no RPE cell migration, less RPE overgrowth, less retinal capillary proliferation, and less retinal disorganization.

Based on current imaging studies, including optical coherent tomography (OCT), common features of CHRRPE lesions include tumor involvement of the internal and intermediate layers of the retina (the inner layer of the retina to the outer plexus layer) with varying amounts of thickened gray-white retinal tissues and epiretinal membranes, the displacement of the surrounding retina and blood vessels, no choroid involvement $[6,7]$.

In 2018 Dedania et al. proposed a new clinical classification system for the assessment and follow-up of patients with CHRRPE. The use of a uniform classification system is intended to facilitate the assessment and comparison of the results of various tests, to determine the frequency of follow-up visits and/or to take therapeutic measures. Changes are classified based on location, fundus features and OCT.

Location of the lesion: zone $1-$ macula/per-macular zone, zone 2 - medium retinal periphery, zone 3 - distal retinal periphery.

Retinal assessment during fundoscopy: stage 1 - no retinal vitreous traction, stage 2 - the presence of retinal traction and/or dissection, stage 3 - retinal detachment.

Retinal morphology in OCT: A - epiretinal membrane, $\mathrm{B}$ - changes in the deeper layers of the retina, and $\mathrm{C}$ - changes on the entire retinal thickness and in RPE.
In patients under 12 years, a full ophthalmologic and morphological assessment is recommended at least every 6 months, with more frequent observation (2-4 months) in patients with macular/perimacular lesions (zone 1) or with retinal traction, dissection or detachment retina (stages 2 and 3). Surgical intervention is recommended in patients with deterioration or loss of vision secondary to retinal vitreous traction or retinal detachment. Routine assessment in patients with lesions in zone 3 is less common, every 6 or every 12 months, depending on the stage of the change [8].

There are no known risk factors. The Caucasian breed seems to dominate. As for gender, in the study by Schachat et al. men and women were equally affected (31 men and 29 women) [9]. However, in the article Shields et al. the majority of patients were men $(68 \%)$.

Shields et al. report the most common presenting signs were decreased vision (40\%) and strabismus $(28 \%)$ in 79 eyes with CHRRPE in a retrospective analysis. The mean vision (logMAR) for the macular tumors' location (51\%) was 1.2, compared with 0.61 in patients with extramacular tumors location (49\%). At 4 years follow-up, visual acuity loss of 3 Snellen lines or more appeared in $60 \%$ of patients with macular CHRRPE, compared with $13 \%$ of peripheral CHRRPE [10]. The visual acuity loss and strabismus are the most frequent presenting signs as in our patient.

CHRRPE are usually unilateral changes. Bilateral lesions are more commonly associated with phacomatoses, including NF type 1, NF type 2, tuberous sclerosis and basal cell nevus syndrome $[11,12]$. Although CHRRPE is more common in patients with NF type 2 , in practice these changes are more commonly seen in patients with NF type 1 due to the higher incidence of NF1. [11] It is recommended to perform genetic tests in patients diagnosed with CHRRPE, especially in patients with binocular presence of characteristic lesions. In hamartomic differentiation, retinoblastoma, melanoma, RPE adenoma, RPE adenocarcinoma should be considered.

\section{CONCLUSIONS}

Classical clinical symptoms, as a painless loss of vision, combined with fundoscopy and diagnostic imaging using optical coherence tomography, allow diagnosis of the disease. The highest risk of blindness or loss of vision are in the presence of CHR- 
RPE with macular/perimacular region involvement with simultaneous retinal vitreous traction, retinal detachment or dissection.

Assessment of anatomy and morphology of the lesion allows it to be properly classified. This facilitates observation of the patient, determining the time interval between subsequent controls and the inclusion of early therapeutic intervention that can protect against vision loss.

\section{REFERENCES}

1. Shields CL, Manalac J, Das C, et al. Review of spectral domainenhanced depth imaging optical coherence tomography of tumors of the retina and retinal pigment epithelium in children and adults. Indian J Ophthalmol. 2015; 63(2): 128-132, doi: 10.4103/0301-4738.154384, indexed in Pubmed: 25827543.

2. Chawla R, Kumar V, Tripathy K, et al. Combined Hamartoma of the Retina and Retinal Pigment Epithelium: An Optical Coherence Tomography-Based Reappraisal. Am J Ophthalmol. 2017; 181: 88-96, doi: 10.1016/j.ajo.2017.06.020, indexed in Pubmed: 28669779.

3. Nowomiejska K, Zarnowski T, Rejdak R, et al. Combined Hamartoma of the Optic Disc and Retinal Pigment Epithelium - 3 Years of the Follow-Up with Semi-Automated Kinetic Perimetry. Open J Ophthalmol. 2012; 02(04): 116-118, doi: 10.4236/ojoph.2012.24025.

4. Gass JD. An Unusual Hamartoma of the Pigment Epithelium and Retina Simulating Choroidal Melanoma and Retinoblastoma, "Transactions of the American Ophthalmological Society. Transactions Am Ophthalmol Soc. 1973; 71: 175-183.
5. Agarwal A. Gass' atlas of macular diseases. Vol. 1. Elsevier, St. Louis 2012. . http://site.ebrary.com/id/10521767EW. (2015 Feb 8).

6. Arepalli S, Pellegrini M, Ferenczy SR, et al. Combined hamartoma of the retina and retinal pigment epithelium: findings on enhanced depth imaging optical coherence tomography in eight eyes. Retina. 2014; 34(11): 2202-2207, doi: 10.1097/IAE.0000000000000220, indexed in Pubmed: 25102194.

7. Shields CL, Thangappan A, Hartzell K, et al. Combined hamartoma of the retina and retinal pigment epithelium in 77 consecutive patients visual outcome based on macular versus extramacular tumor location. Ophthalmology. 2008; 115(12): 2246-2252.e3, doi: 10.1016/j. ophtha.2008.08.008, indexed in Pubmed: 18995912.

8. Dedania VS, Ozgonul C, Zacks DN, et al. ovel classification system for combined hamartoma of the retina and retinal pigment epithelium. Retina. 2018; 38(1): 12-19, doi: 10.1097/IAE.0000000000001499, indexed in Pubmed: 28098730.

9. Schachat AP, Shields JA, Fine SL, et al. Combined hamartomas of the retina and retinal pigment epithelium. Ophthalmology. 1984; 91(12): 1609-1615, doi: 10.1016/s0161-6420(84)34094-5, indexed in Pubmed: 6521994.

10. Shields CL, Thangappan A, Hartzell K, et al. Combined hamartoma of the retina and retinal pigment epithelium in 77 consecutive patients visual outcome based on macular versus extramacular tumor location. Ophthalmology. 2008; 115(12): 2246-2252.e3, doi: 10.1016/j. ophtha.2008.08.008, indexed in Pubmed: 18995912.

11. Mason JO, Kleiner R. Combined hamartoma of the retina and retinal pigment epithelium associated with epiretinal membrane and macular hole. Retina. 1997; 17(2): 160-162, indexed in Pubmed: 9143046.

12. Kaye $L D$, Rothner $A D$, Beauchamp GR, et al. Ocular findings associated with neurofibromatosis type II. Ophthalmology. 1992; 99(9): 1424-1429, doi: 10.1016/s0161-6420(92)31789-0, indexed in Pubmed: 1407974. 